fectious Diseases. Infectious agents surveillance report: flash report of influenza virus in Japan, 2010/11 season. http:// idsc.nih.go.jp/iasr/influ-e.html. Accessed April 5, 2011.

8. Marcel JP, Alfa M, Baquero F, et al. Healthcare-associated infections: think globally, act locally. Clin Microbiol Infect 2008;14: 895-907.

\section{Infection Control Implications of Nosocomial Pertussis Exposure in a Hospitalized Adult during Influenza Season}

To the Editor-During influenza season, some disorders present as influenza-like illnesses (ILIs) and may mimic influenza (eg, human parainfluenza virus 3 infection and Legionnaires' disease).$^{1-3}$ At our hospital during this influenza season, influenza in hospitalized adults has been due to human seasonal influenza $A(\mathrm{H} 3 \mathrm{~N} 2)$ and swine influenza $A(\mathrm{H} 1 \mathrm{~N} 1)$ virus. Recently, an elderly woman with a history of chronic bronchitis was admitted to our hospital for an ILI. Her chief complaint was persistent dry cough. Although the working diagnosis was influenza, the patient was not placed under influenza (droplet) precautions or treated with oseltamanivir. Although pertussis was considered in the differential diagnosis and pertussis diagnostic tests were ordered, the patient was not placed under droplet precautions for potential pertussis. When polymerase chain reaction performed on a nasopharyngeal swab sample obtained from the patient was reported to have results that were positive for Bordetella pertussis on hospital day 3,51 healthcare workers and the patient's roommate had already been exposed to potential infection.

A 71-year-old woman presented to our institution with a nonproductive cough. Her illness had begun with nasal congestion approximately 1 week before presentation. The patient then developed a nonproductive cough with some shortness of breath. She denied fever, chills, nausea, vomiting, diarrhea, or myalgias. She reported having recent contact with her grandchildren, who had upper respiratory tract infections. Her medical history was remarkable for chronic bronchitis, diabetes mellitus, systemic lupus erythematosus, heart failure, and osteoarthritis. She had quit smoking 11 years earlier.

At admission to the hospital, the patient was afebrile with a pulse of 92 beats $/ \mathrm{min}$, blood pressure of $122 / 45 \mathrm{~mm} \mathrm{Hg}$, and a respiratory rate of 15 breaths/min. She was in no distress but was constantly coughing. Her physical examination had findings that were within normal limits except for bilateral rhonchi with wheezing and mild lower-extremity edema. Her initial white blood cell count was 7,500 cells $/ \mathrm{mm}^{3}$ (69\% neutrophils, $19 \%$ lymphocytes, $9 \%$ monocytes, and $2 \%$ eosinophils), and her platelet count was 184,000 cells $/ \mathrm{mm}^{3}$. Her serum transaminase and ferritin levels were normal, but her erythrocyte sedimentation rate of $82 \mathrm{~mm} / \mathrm{h}$ (normal range, $1-35 \mathrm{~mm} / \mathrm{h}$ ) was highly elevated. Chest radiographs obtained at admission had normal findings. To treat possible community-acquired pneumonia, the patient initiated a regimen of ceftriaxone ( $1 \mathrm{~g}$ administered intravenously every 24 hours), azithromycin ( $500 \mathrm{mg}$ administered intravenously every 24 hours), and methylprednisolone. Her rapid influenza test result was negative, and her nasopharyngeal respiratory viral fluorescent antibody panel had results that were negative for influenza A and B virus, parainfluenza viruses, respiratory syncytial virus, adenovirus, and metapneumovirus. Her Chlamydophila pneumoniae and Mycobacterium pneumoniae immunoglobulin (Ig) M titers were not elevated. B. pertussis IgM titer was highly elevated at $2.3 \mathrm{U} / \mathrm{mL}$ (normal range, $0.0-0.9 \mathrm{U} / \mathrm{mL}$ ), and her $B$. pertussis IgG titer was $4 \mathrm{U} / \mathrm{mL}$ (normal range, $0-9 \mathrm{U} / \mathrm{mL}$ ).

Pertussis, which is caused by $B$. pertussis, is most common in childhood but is not uncommon among elderly adults. Unlike classic pertussis in children, adult pertussis presents as a persistent nonproductive cough that lasts for several weeks. Pertussis pneumonia, although less common among adults than among children, may also occur. ${ }^{4-8}$ In adults hospitalized with ILIs, pertussis may go unnoticed or undiagnosed. Because pertussis is highly contagious and is spread via droplets, the potential for nosocomial spread among hospitalized patients with pertussis is great. ${ }^{9,10}$ At hospital admission, patients with suspected or known pertussis should be placed under droplet precautions and treated with a macrolide for 5 days. After macrolide therapy, coughing often persists, but the contagious potential of pertussis should be diminished.

This case of pertussis in a hospitalized adult during influenza season is emblematic of a common infection control problem. Although her physicians suspected pertussis, they did not place the patient under droplet precautions until the nasopharyngeal pertussis polymerase chain reaction result for the swab sample was reported 3 days after the hospital admission. During this 72 -hour period, there were 51 healthcare workers with potential exposure ( 8 of whom had close contact exposures). The patient's roommate was also exposed to pertussis for 3 days and was given azithromycin prophylaxis.

This case underscores important epidemiologic and infection control lessons. First, although every ILI in a hospitalized adult during influenza season is not due to influenza virus, adults who are admitted to the hospital with ILIs should be placed under influenza (droplet) precautions at hospital admission pending diagnostic testing for influenza. Second, if pertussis, which is a highly contagious infectious disease, is in the differential diagnosis, the patient should be under appropriate droplet precautions pending test results to decrease the potential for nosocomial pertussis. Although influenza was the working diagnosis in this case, there would have been no nosocomial pertussis exposures because droplet precautions are appropriate for pertussis as well as for influenza. The clinical presentation of adult pertussis may mimic an ILI or influenza with dry cough, leukocytosis, and no infiltrates 
TABLE 1. The Differential Diagnostic Features of Pertussis and Influenza A in Adults

\begin{tabular}{lcc}
\hline & Pertussis & Influenza A \\
\hline Symptoms & & + \\
Dry cough & + & - \\
Prolonged cough $(>1$ week $)$ & \pm & - \\
Hoarseness & - & + \\
Myalgias & & \\
Signs & - & + \\
Temperature $>38^{\circ} \mathrm{C}$ & - & \pm \\
Conjunctival suffusion & & + \\
Laboratory tests & + & + \\
Leukocytosis & - & + \\
Relative lymphopenia & - & \pm \\
Lymphocytosis & - & - \\
Thrombocytopenia & - & + \\
Serum transaminase level & + & \\
Elevated erythrocyte sedimentation rate $(>50 \mathrm{~mm} / \mathrm{h})$ & & - \\
Chest film findings & + & \pm \\
No infiltrates & & \\
Perihilar infiltrates & & \\
Increase in basal lung markings/atelectasis & & \\
\hline
\end{tabular}

visible on chest radiographs, and it may not be accompanied by leukocytosis or lymphocytosis. Leukopenia and thrombocytopenia are not features of adult pertussis (Table 1). Third, although this patient had received pertussis vaccination in childhood, protection against pertussis lasts for only 10 years.

As with influenza, patients with an ILI or suspected pertussis should be put under appropriate precautions (ie, droplet precautions) when admitted to the hospital, rather than when test results are reported. Because childhood pertussis vaccination does not confer lifelong immunity, revaccination of adults who are at high risk (eg, healthcare workers) with tetanus, diphtheria, and pertussis vaccine (Tdap) is currently recommended. Of the 51 healthcare workers with exposure to pertussis, 8 were in close contact with the coughing patient. Six of the 8 healthcare workers who had close contact with the patient had received Tdap during the past 4 years, and all 8 were given azithromycin prophylaxis for 5 days. There were no secondary pertussis cases in any of the 51 exposed healthcare workers. Also, the patient's roommate did not develop pertussis.

\section{ACKNOWLEDGMENTS}

Potential conflicts of interest. All authors report no conflicts of interest relevant to this article.

Burke A. Cunha, MD; ${ }^{1}$

Nardeen Mickail, MD; ${ }^{1}$

Valsamma Thekkel, RN, CIC;

Carol Cohan, $\mathbf{R N}^{1}$

Affiliations: 1. Infectious Disease Division, Infection Control Section, Em- ployee Health Section, Winthrop-University Hospital, Mineola, New York, and State University of New York School of Medicine, Stony Brook, New York.

Address correspondence to Burke A. Cunha, MD, Chief, Infectious Disease Division, Winthrop-University Hospital, Mineola, NY 11501 (llusardi@winthrop.org).

Infect Control Hosp Epidemiol 2011;32(8):826-827

(C) 2011 by The Society for Healthcare Epidemiology of America. All rights reserved. 0899-823X/2011/3208-0017\$15.00. DOI: $10.1086 / 661223$

\section{REFERENCES}

1. Cunha BA. Swine influenza (H1N1) pneumonia: clinical considerations. Infect Dis Clin North Am 2010;24:203-228.

2. Cunha BA, Klein NC, Strollo S, et al. Legionnaires' disease mimicking swine influenza $(\mathrm{H} 1 \mathrm{~N} 1)$ pneumonia during the "herald wave" of the pandemic. Heart Lung 2010;39:242-248.

3. Cunha BA, Corbett $M$, Mickail N. Human parainfluenza virus type 3 (HPIV 3) viral community-acquired pneumonia (CAP) mimicking swine influenza (H1N1) during the swine flu pandemic. Heart Lung 2011;40:76-80.

4. Morse SI. Pertussis in adults. Ann Intern Med 1968;68:953-954.

5. Wright SW, Edwards KM, Decker MD, et al. Pertussis infection in adults with persistent cough. JAMA 1975;273:1044-1046.

6. Linnemann CC Jr, Nasenbeny J. Pertussis in the adult. Annu Rev Med 1977;28:179-185.

7. Robertson PW, Goldberg H, Jarvie BH, et al. Bordetella pertussis infection: a cause of persistent cough in adults. Med J Aust 1987; 146:522-525.

8. Herwaldt LA. Pertussis in adults: what physicians need to know. Arch Intern Med 1991;151:1510-1512.

9. Kurt TL, Yaeger AS, Guenette S, et al. Spread of pertussis by hospital staff. JAMA 1972;221:264-267.

10. Weber DJ, Rutala WA. Management of healthcare workers exposed to pertussis. Infect Control Hosp Epidemiol 1994;15: 411-415. 